\title{
Materials Comparison of 3D Printed Scaffolds for Bone Tissue Engineering Applications
}

\author{
Ling Zhao ${ }^{1}$, Lihua Liang ${ }^{2, *}$ \\ ${ }^{1}$ School of Stomatology, Guilin Medical University, Guilin, Guangxi, China \\ ${ }^{2}$ Department of Prosthodontics, Affiliated Stomatology Hospital, Guilin, Guangxi, China \\ *Correspondence Author, llh7907@163.com
}

\begin{abstract}
In recent years, 3D printed bone tissue engineering scaffolds have developed rapidly in the field of bone defect repair, and their materials research, development and preparation are very prospective, with a significant long-term clinical application effect, which is expected to solve further the problems of vascularization disorder, osteogenesis difficulty and cell activity maintenance during the bone regeneration process. The application advancement of frequently used $3 D$ printing bone scaffold materials is presented in this review by extracting relevant literature from each journal database and merging it with current research. The application effects of different materials are compared to improve the standard and process specification of $3 D$ printing materials and further enhance the recognition and satisfaction of clinical treatment.
\end{abstract}

Keywords: 3D printing, Bone scaffold, Bone defect, Bone regeneration.

\section{Introduction}

Along with the advances in medical regeneration and tissue engineering technology, the goal of bone defect repair has gradually changed from replacing the function of the prosthesis to inducing the regeneration of the organism, prompting the development of bone tissue engineering scaffolds with 3D printing technology as the core to be more widely used. In the light of recent research advances in 3D printing technology for bone regeneration in the medical field, we compare the performance advantages of various materials used to prepare bone scaffolds.

Due to the complexity of bone tissue structure, the synthesis process of ideal bone scaffolds must be excellent, and the selection of preparation materials is also very demanding. Its biocompatibility, osteogenic induction, morphological plasticity, controlled degradability, mechanical properties, and other properties are key factors affecting bone tissue healing and regeneration[1]. At present, the widely used 3D printing materials are mainly metal, inorganic non-metal, polymer substances and other materials[2]. This article reviews and analyzes the current progress in applying related materials of 3D printed bone tissue engineering scaffolds and looks forward to the development trend of 3D printing technology in the field of bone defect repair in the future.

\section{Metallic Materials}

Metallic materials are among the most common 3D printing materials in clinical promotion because of their excellent mechanical stability, conductivity, and flexibility [3], which can repair bone tissue defects. Among them, synthetic metal materials dominated by titanium and magnesium have become increasingly mature in the medical application of $3 \mathrm{D}$ printing technology.

\subsection{Titanium and Titanium Alloys}

Titanium and titanium alloys are widely used in treating clinical bone defects due to their advantages of light quality, strong corrosion resistance, high mechanical properties, and good biocompatibility[4]. Due to the jaw's complex anatomy and individual differences, it is challenging to prepare synthetic metal materials such as titanium mesh and titanium nails by relying on traditional processes. In contrast, a variety of personalized repair methods of bone defects by titanium and its alloys can be realized using 3D printing technology. At present, it has been widely used in maxillofacial surgery, tooth defect repair and other treatment[5]. Kownacki Patryk et al.[6] performed alveolar ridge reconstruction in 5 patients with severe mandibular defects by $3 \mathrm{D}$ printed personalized titanium scaffolds. CBCT examination showed that the clinical effect was good, but the early exposure of titanium scaffolds affected the healing process of patients. Therefore, the preparation of bone tissue engineering scaffolds with reasonable spatial structure, good mechanical properties and biocompatibility by 3D printing technology has high practical significance. Chanchareonsook et al.[7] prepared Ti-6Al-4V titanium alloy scaffold by $3 \mathrm{D}$ printing for osteogenic induction in the rhesus monkeys of mandibular defect also achieved a good repair effect. Due to the low bonding strength between traditional titanium alloy and bone tissue, it is easy to cause stress shielding after implantation in vivo. Xu Sun et al.[8] formed graphene coating on the surface of titanium alloys by the micro-arc oxidation process, which improved the surface roughness of titanium alloys. The experimental results of physical and chemical properties showed that the compressive strength and elastic modulus of this $3 \mathrm{D}$ printed porous graphene-titanium alloy bone scaffolds were matched with normal bone tissue and had good repair potential. Chunqiu Zhang et al.[9] prepared bone-structured titanium alloys using 3D printing technology based on electron beam melting (EBM), improved the mechanical properties of titanium alloys, made the elastic modulus and mechanical strength of the scaffolds close to those of normal bones effectively reduced stress shielding. The porous titanium alloy scaffolds prepared by Linwei Lyu et al.[10] using 3D printing technology has a better effect on early postoperative osteogenesis induction. However, long-term postoperative bone tissue growth's morphological structure and chemical composition still lack in-depth study. 


\subsection{Magnesium and Magnesium Alloys}

In recent years, magnesium metal materials have been considered as one of the biodegradable, stable mechanical properties and bone-inducing 3D printing materials, which have broad application prospects in the field of bone regeneration. Its implantation can release Magnesium ions and further stimulate new bone formation, while porosity becomes an essential determinant for the safe and effective implantation of magnesium scaffolds in vivo. By implanting cell-loaded porous magnesium alloy scaffolds into the mandibular defect area of animals, Wang et al.[11] found that hemolysis didn't occur in the animals. They confirmed that the scaffolds were biocompatible and non-cytotoxic, with long-lasting effects on animal organ protection. Dong et al.[12] studied a 3D printing method for preparing porous magnesium scaffolds based on topological sorting at room temperature. Through loading magnesium powder, solvent casting 3D printing (SC-3DP), stripping and sintering, layered porous magnesium scaffolds with high porosity. TGA, FTIR, SEM and other experimental results showed that the scaffolds had high fidelity and good compactness. Thus excellent characteristic properties provided more possibilities for bone growth and induced osteogenesis.

The above metal materials have good biocompatibility, superior mechanical properties and controllable degradation. However, these metal materials need to be printed at high temperatures, and their long-term clinical effect has not been recognized, which urgently needs further study and solution.

\section{Inorganic non-metallic Materials}

Bioceramics are the most used inorganic non-metallic bone repair materials in bone tissue engineering, and their main components are similar to those of human bone inorganic materials. The main properties include osteoconductivity, aesthetics, biocompatibility, chemical stability and controllable degradation. The 3D printing method of laser sintering can also be applied to thoroughly compound adhesives with ceramic powders to improve the accuracy of scaffold material preparation. Bioceramic materials for 3D printed bone tissue engineering scaffolds can be divided into calcium apatite and bioactive glass.

\subsection{Calcium Apatite}

Calcium apatite is a critical component of human bone growth. Its common types in the clinical application are hydroxyapatite (HA) and $\beta$-tricalcium phosphate ( $\beta$-TCP). Hydroxyapatite (HA) is the main inorganic component of human bone tissue with good biological activity, cell compatibility and bone conductivity, but it is brittle and slow to degrade. $\beta$-tricalcium phosphate $(\beta$-TCP) has good biological degradability, biological compatibility and antibacterial properties, but its mechanical strength is low, and its degradation is too fast in the physiological environment. Therefore, they are often mixed to make up for the lack of single materials and provide advantages for applying bone tissue engineering. Yanan Wang et al.[13] compared the effect of different HA and $\beta$-TCP compositions on the mechanical properties of their bone scaffolds and found that the compressive strength of the scaffolds increased with the increase of HA and $\beta$-TCP weight ratio. Scanning electron microscopy showed that the size of HA and $\beta$-TCP particles determined the size of micro-pores. They also proved that the optimal weight ratio of HA/ $\beta$-TCP scaffolds with superior mechanical properties and biological characteristics prepared by $3 \mathrm{D}$ printing was $60: 40$. This material might be the best combination for bone tissue engineering applications. In addition, due to the high fragility of such materials, it is often combined with some polymer co-printing in the practical application of preparing bone scaffolds, and Smeets et al.[14] $3 \mathrm{D}$ printing technology based on selective laser melting (SLM) to prepare porous polypropylene ester/ $\beta$-tricalcium phosphate composite scaffolds to repair the rat models of skull defects, and observed that the osteogenesis performance of the scaffolds group was better than that of the autologous bone repair group at 30 days after surgery. Sun et al.[15] developed a tricalcium phosphate/Poly(lactic-co-glycolic acid) scaffolds by $3 \mathrm{D}$ printing, and introduced bone morphogenetic protein into the scaffolds, and then implanted the scaffold into animals. The results showed that the osteogenic differentiation effects of the scaffolds in animals were obvious, which further confirmed that the composite of calcium apatite and synthetic polymer could magnify the advantages of each component.

\subsection{Bioactive Glass}

Bioactive glasses (BGs) are mainly composed of silicate and phosphate. Their interconnected porous structure is similar to bone, which has good osteoconductivity and provides a temporary renewable template for the cells[16]. A comparative study of $45 \mathrm{~S} 5$ bioactive glass and autologous iliac crest transplantation in treating adolescent idiopathic scoliosis[17] showed that the osteogenic induction of bioactive glass was as effective as iliac crest transplantation and less postoperative complications. Although BGs have excellent biological activity and bone induction, their inherent brittleness has become a potential limitation for clinical application. Biodegradable polymers should be added to synthesize BGs composites to improve porosity, degradation rate and elastic modulus. Researchers have developed mesoporous bioactive glasses (MBGs) scaffolds by adding additional oxides into BGs and using 3D printing assisted CAD/CAM technology[18]. Compared with traditional bioactive glasses, the implantation effect of MBGs has better osteogenic performance, degradation performance, mechanical properties and mechanical strength. It reduces the potential tissues, which supports the induction of bone tissue regeneration. However, the preparation of MBGs has not yet been standardized and needs further research.

The bioceramic materials mentioned above have remarkable osteogenic induction, compressive strength and other repair properties. Still, their printing conditions are similar to metal materials, which must be prepared at high temperatures. Therefore, improving the preparation conditions will be a breakthrough for the successful development of bioceramic bone tissue scaffolds in the future.

\section{Bioinks with Cell Involvement}

At present, metal and inorganic non-metal materials are widely used in the field of bone defect repair, the curative 
effect is feasible, but the rejection of the body to the allogeneic tissue still exists. Therefore, in order to achieve the biocompatibility of autologous transplantation, it is necessary to prepare bone tissue engineering scaffolds with cell activity and biocompatibility, which has become the new development trend of $3 \mathrm{D}$ printing. The development of biological ink with excellent characteristics such as printability, structural stability, biodegradability and cell activity has also become the main difficulty for transforming 3D biological printing technology into clinical application. To overcome this difficulty, David Chimene et al.[19] developed a nanoengineered ionic covalent entanglement (NICE) bioink formulation suitable for 3D bioprinting. The bioink was remodelled by degradation test and histologically tested after three months. They found that the bone-like matrix of collagen and proteoglycan could be deposited in the early stage of cell culture, then carbonate and phosphate could prolong the mineralization as well as calcium in the later stage. The final SEM-EDS data and calcium analysis results proved that NICE biological ink could accurately print, mechanical properties, and biological degradability. Even without bone morphogenetic protein 2 (BMP-2), NICE biological ink could induce human mesenchymal stem cells (hMSCs) to differentiate into bone, which played an essential role in guiding bone tissue reconstruction.

The grid structure formed by the extracellular matrix (ECM) in the human body plays a supporting role in maintaining the body's homeostasis and regulating normal life activities and is one of the ideal bioink materials at present. Kang et al.[20] constructed 3D printing bone scaffolds using human articular cartilage ECM to obtain an acellular extracellular matrix (AECM). This network scaffold is more conducive to the adhesion and proliferation of mesenchymal stem cells to provide mechanical support for cartilage tissue regeneration. It is rich in type I collagen and can be used as a heterogeneous nucleation template to induce calcium and phosphorus, which is excellent for bone regeneration. However, its dense fibrous structure decreases cell permeability and becomes a constraint obstacle for bone scaffolds. Yunxiang Luo et al.[21] generated tendon extracellular matrix (tECM) and combined it with polyethene glycol diacrylate (PEGDA). They used stereolithography (SLA) technology to form 3D printed polyporous PEGDA/tECM scaffolds (3D-pPES). Their observation showed that the 3D-pPES group had higher cell migration efficiency and osteogenic differentiation RNA level, which powerfully indicated that this bioink had better repair and degradation performance.

\section{Polymer Materials}

Polymers mainly include natural polymers and synthetic polymers. For natural polymer, the polymers commonly used in bone tissue engineering are alginate, collagen, silk fibroin, chitosan, etc. Because of their excellent water-solubility, but defects such as weak mechanical strength and difficulty in massive preparation[22]. At present, the most common synthetic polymers used in clinical practice are polylactic acid (PLA), polycaprolactone (PCL), polyetheretherketone (PEEK), photosensitive resin, etc. These synthetic materials have good mechanical strength and degradation ability and can be synthesized and output in large quantities[23]. The following four synthetic polymer materials are described in detail.

\subsection{Polylactic Acid}

Polylactic acid (PLA) has good thermal processability, tensile strength, elastic modulus and biocompatibility. Bone tissue engineering is usually used as a matrix component to prepare cell-compatible bone scaffolds after compounding with hydroxyapatite (HA). Ikumi $\mathrm{R}$ et al.[24] prepared hydroxyapatite/poly-L-lactic acid (HA/PLLA) composite scaffolds by $3 \mathrm{D}$ printing and implanted them in a rat model with skull defects for observation, demonstrating that PLLA combined with HA is more effective in promoting new bone formation. Du et al.[25] printed PCL/HA composite bone branches and pure PCL material bone scaffolds by selective laser sintering (SLS) technique and implanted them into animals. It is concluded that the composite scaffolds can better promote cell proliferation and improve the ability of osteogenic induction. Zhou et al.[26] prepared polylactic acid porous cylinders, porous spiral and solid spiral scaffolds with three different morphological structures by 3D printing. At the same time, human fetal osteoblasts (hFOB) were inoculated on three scaffolds to observe the morphological changes of cells. After 28 days of culture, they found that the proliferation of hFOB cells on porous scaffolds was significantly greater than that of solid spiral scaffolds. The porous structure of porous spiral scaffolds was more conducive to inducing cell growth. In addition, ALP activity and mineral deposition indicated that the porous spiral scaffold stimulated new bone formation better than the other two. Therefore, the porous spiral scaffold can realize the connection of the porous network inside the scaffold, which significantly affects cell proliferation and differentiation and can repair severe bone defects.

\subsection{Polycaprolactone}

Polymeric lactone (PCL) has better biocompatibility, higher mechanical properties and load application potential compared with other polyesters. The slow degradation of PCL is beneficial to bone remodelling, thereby regulating the biodegradation rate of polymers and preparing bone repair scaffolds. In addition, due to its melting temperature of $55-60^{\circ} \mathrm{C}$, it has become one of the preferred polymers for $3 \mathrm{D}$ printing extrusion. However, a single PCL has no potential to induce osteogenesis and needs to be combined with various polyesters, inorganic substances, metal elements, or collagen to improve the performance of the scaffolds. It has been shown that improving the pore size of 3D printed PCL scaffolds can effectively promote new bone. Min-Chia Chen et al.[27] implanted 3D-printed $900 \mathrm{~mm}$ (aperture) PCL scaffold into the skull defect of rats. The X-ray images showed that the mineralization of the bone defect in the scaffold group was gradually apparent; histology showed that the new bone in the scaffold group grew to the centre of the bone defect, while the non-scaffold group slowly formed bone along the defect edge. Their experimental results indicate that the PCL scaffold with a 3D printing aperture of $900 \mu \mathrm{m}$ had the potential to promote the formation of new bone. The new bone didn't closely contact the scaffolds, so the affinity between bone tissue cells and PCL scaffolds needed to be improved. Luo et al.[2] prepared poly-caprolactone/ quaternary salt/oyster shell powder scaffold by $3 \mathrm{D}$ printing. 
The results of SEM, FTIR, mechanical test and control experiment showed that the scaffolds had antibacterial properties, high mechanical stability, good bone induction and good biocompatibility. The preparation raw materials were rich in sources, easy to obtain and had little cost. At present, most of the in vivo studies of PCL stents stay in animal trials, no human clinical trials have been conducted[28], and their long-term efficacy has not been confirmed. Therefore, PCL-based tissue engineering still has unlimited potential in the future.

\subsection{Polyetheretherketone}

Polyetheretherketone (PEEK) is an artificial synthetic polymer material with high physical properties and excellent biocompatibility, which can be immersed in a body fluid environment for a long time[29]. Scolozzi et al.[30] produced PEEK bone scaffolds through 3D printing technology to repair patients with unilateral skull and mandibular defects. The postoperative feedback effect of patients was significant, but the long-term impact has not been confirmed. Deng et al.[31] developed a PEEK/Ag composite bone scaffold suitable for $3 \mathrm{D}$ printing through the catechin amine chemical method. The bacterial kinetics and antibacterial membrane test showed that the scaffolds modified with Ag coating had a strong antibacterial effect. The ALP activity test showed higher cell activity than pure PEEK scaffolds. Feng et al.[32] studied the mechanical and biological characteristics of porous PEEK scaffolds with different pore sizes in vitro and vivo. The experimental results in vitro and in vivo support that porous PEEK scaffolds have good biocompatibility. The porous structure is beneficial to cell adhesion, proliferation and osteogenic differentiation. It can promote the preferential potential of bone growth and vascular perfusion, and the pore size of $450 \mu \mathrm{m}$ is the best. For example, the successful repair cases of PEEK mentioned above can be known that compared with metal materials such as titanium alloy, PEEK has closer elastic modulus and mechanical strength to natural bone, which can effectively reduce the risk of stress shielding and loosening after implantation. However, many studies have reported that PEEK hasn't a biological activity, and its bone conduction ability is meagre. It is urgent to further surface modification to give more biological characteristics.

\subsection{Photosensitive Resin}

The photosensitive resin is mainly composed of the prepolymer, reactive diluent, photoinitiator and filler, which has the advantages of good fluidity, excellent thermal stability, superior gloss and slight cytotoxicity. However, it will shrink in the curing reaction, reduce the preparation accuracy, and easily cause deformation and distortion of 3D printing scaffolds. Ming-You Shie et al.[33] used hyaluronic acid-based waterborne polyurethane as the surface coating material of photosensitive resin to prepare 3D printing composite scaffolds. Human Wharton's jelly mesenchymal stem cells (hWJMSCs) were seeded on the scaffolds and cultured. They found that the 3D composite scaffold had good biocompatibility and effectively promoted bone regeneration. In recent years, researchers also favour photosensitive resin in $3 \mathrm{D}$ printing applications. The accuracy and structural design of photosensitive materials are expected to be improved.

\section{Composite Materials}

It is necessary to make up for the defects of various $3 \mathrm{D}$ printing materials to achieve a perfect $3 \mathrm{D}$ printed scaffold in bone tissue engineering. Two or more substances with different physical and chemical properties are combined into composite materials through appropriate methods to facilitate the research and production of bone scaffolds with excellent performance. Richard et al.[34] found that the control group with single polymer scaffold material would cause a decrease in the number of peripheral neovascularization after one month of implantation. In contrast, the experimental group of a composite polymer scaffold containing bioactive glass was significantly increased, which effectively explained the role of composite materials in bone induction. The bone scaffold printing materials formed by polymer and bioceramics are similar to natural bone, recognized by the academic community and widely used in bone tissue engineering. Tu et al.[35] researched porous composite scaffolds of polylactic acid and hydroxyapatite with a ratio of $4: 1$ by 3D printing. In vivo and in vitro experiments showed that $3 \mathrm{D}$ printed PLA/HA porous composite scaffolds had excellent biocompatibility and high mechanical strength. This study confirmed the advantages of composite materials and provided an economical and effective method for bone tissue engineering scaffolds. Since the antibacterial properties of synthetic polymers are primarily poor, the infection of the implanted sites of scaffolds prepared by them is considered the main reason for delayed bone healing and failure of treatment. Socrates Radhakrishnan et al.[36] reduced silver nanoparticles (AgNps) in polycaprolactone (PCL) solution and extruded them into PCL/AgNps filaments. The scanning electron microscope showed that the 3D printing PCL/AgNps scaffolds had interconnected porous structures. The X-ray photoelectron spectroscopy analysis showed that PCL/AgNps scaffolds could enhance the stiffness and antibacterial properties.

In addition, the composite materials can combine with cytokines, growth factors and other active factors with osteogenic induction during printing to further enhance the guiding effect of $3 \mathrm{D}$ printing bone scaffolds on the formation of new bone. Researchers have also obtained excellent bone scaffold printing materials using biological bionic structures. Inspired by the unique biological design of "Lotus", Han et al.[37] researched 3D printed porous bioceramic scaffolds with "Lotus" bionic structure, containing deferoxamine (DFO) liposomes as "Lotus". In vitro experiments showed that the composite scaffolds effectively promoted the formation of vascular morphology of human umbilical vein endothelial cells (HUVEC). In vivo experiments showed that scaffolds could enhance vascularization of bone tissue and induce the expression of bone-related proteins Hif1- $\alpha$, CD31, OPN and OCN. This bionic structure inspired 3D printing internal vascularization bone scaffolds considerably shortened the bone repair time. Therefore, 3D printing technology can use various materials with different physical and biological properties and introduce active ingredients in vivo to print to realize the complementary advantages of each material component and achieve the best clinical application effect. 


\section{Summary and Outlook}

In summary, composite materials have more advantages than single materials. They can meet the performance requirements that cannot be achieved by single materials, such as excellent biocompatibility, significant controllable degradation and adequate mechanical strength, which will become the gospel in the field of bone defect repair. Furthermore, Biological ink will also more effectively improve the vascularization and ossification of the body. However, the former international academia has not yet formed a unified standard for developing $3 \mathrm{D}$ printing materials. Its high development cost and complex technology promotion have become the main problems restricting development presently. Therefore, in the future, it is urgent to improve the standards and process specifications of $3 \mathrm{D}$ printing materials to develop and prepare more economical, efficient and safe 3D printed scaffolds in bone tissue engineering, which are widely used in clinical practice and serve the public.

\section{References}

[1] L. Zhang, G. Yang, B.N. Johnson, et al. "Threedimensional (3D) Printed Scaffold and Material Selection for Bone Repair," ActaBiomater, 84, pp. 16-33, 2019.

[2] W. Luo. "Preparation and characterization 3D printing scaffolds for bone tissue engineering," Jinan University, China, 2017.

[3] K. Wang, S. Zheng, S. Pan, et al. "Preparation of 3D printed bone tissue engineering scaffold," Chinese Journal of Tissue Engineering Research, 23 (34), pp. 5516-5522, 2019.

[4] X. Zhang, W. Wu. "Research progress on 3D printing technology and biomaterials for bone reconstruction in maxillofacial regions," International Journal of Stomatology, 47 (06), pp. 677-685, 2020.

[5] Y. Dang, Y. Li, R. Li, et al. "Three-dimensional printing technology preparation of bone tissue engineering scaffold materials in bone defect repair," Chinese Journal of Tissue Engineering Research, 21 (14), pp. 2266-2273, 2017.

[6] K. Patryk, M. Tadeusz, W. Maciej, et al. "Clinical application of titanium individual bone scaffold using 3D printing in the reconstruction of alveolar bone ridge," Clinical Oral Implants Research, 28, pp. 508-508, 2017.

[7] N. Chanchareonsook, H. Tideman, S. Lee, et al. "Mandibular reconstruction with a bioactive-coated cementless Ti6Al4V modular endoprosthesis in Macaca fascicularis," International Journal of Oral \& Maxillofacial Surgery, 43 (6), pp. 758-768, 2014.

[8] X. Sun, S. Tong, S. Yang, et al. "The Effects of Graphene on the Biocompatibility of a 3D-Printed Porous Titanium Alloy," Coatings, 11 (12), pp. 1509, 2021.

[9] C. Zhang, L. Zhang, L. Liu, et al. "Mechanical behaviour of a titanium alloy scaffold mimicking trabecular structure," Journal of Orthopaedic Surgery and Research, 15 (1), pp. 40, 2020.

[10] L. Lyu, S. Yang, Y. Jing, et al. "Examining trabecular morphology and chemical composition of peri-scaffold osseointegrated bone," Journal of Orthopaedic Surgery and Research, 15 (1), pp. 406, 2020.
[11] L. Wang, Y. Guo, H. Huang, et al. "Evaluation of biosafety of porous magnesium alloy scaffolds for jaw defects," Chinese Journal of Tissue Engineering Research, 23 (26), pp. 4121-4128, 2019.

[12] J. Dong, Y. Li, P. Lin, et al. "Solvent-cast 3D printing of magnesium scaffolds," Acta Biomaterialia, 114, pp. 497-514, 2020

[13] Y. Wang, K. Wang, X. Li, et al. "3D fabrication and characterization of phosphoric acid scaffold with a HA $/ \beta$-TCP weight ratio of $60: 40$ for bone tissue engineering applications," PLoS ONE, 12 (4), pp. e0174870, 2017.

[14] R. Smeets, M. Barbeck, H. Hanken, et al. "Selective laser melted fully biodegradable scaffold composed of poly (D, L-lactide) and $\beta$-tricalcium phosphate with potential as a biodegradable implant for complex maxillofacial reconstruction: in vitro and in vivo results," Journal of Biomedical Materials Research Part B Applied Biomaterials, 105 (5), pp. 1216-1231, 2017.

[15] L. Sun, Z. Xiong. "Poly(lactic-co-glycolic acid)/ tricalcium phosphate scaffolds prepared by rapid prototyping technology for the repair of radial defects in rabbits," Chinese Journal of Tissue Engineering Research, 15 (12), pp. 2091-2094, 2011.

[16] G. Turnbull, J. Clarke, F. Picard, et al. "3D bioactive composite scaffolds for bone tissue engineering," Bioactive Materials, 3 (3), pp. 278-314, 2018.

[17] B. Ilharreborde, E. Morel, F. Fitoussi, et al. "Bioactive glass as a bone substitute for spinal fusion in adolescent idiopathic scoliosis a comparative study with iliac crest autograft," Journal of pediatric orthopaedics, 28 (3), pp. 347-351, 2008

[18] F. Baino, E. Fiume, "3D Printing of Hierarchical Scaffolds Based on Mesoporous Bioactive Glasses (MBGs) - Fundamentals and Applications," Materials, 13 (7), pp. 1688, 2020.

[19] D. Chimene, L. Miller, L.M. Cross, et al. "Nanoengineered Osteoinductive Bioink for 3D Bioprinting Bone Tissue," ACS Applied Materials \& Interfaces, 12 (14), pp. 15976-15988, 2020.

[20] H.J. Kang, J. Peng, S.B. Lu, et al. "In vivo cartilage repair using adipose-derived stem cell-loaded decellularized cartilage ECM scaffolds," Journal of Tissue Engineering and Regenerative Medicine, 8 (6), pp. 442-453, 2014.

[21] Y. Luo, H. Pan, J. Jiang, et al. "DesktopStereolithography 3D Printing of a Polyporous Extracellular Matrix Bioink for Bone Defect Regeneration," Frontiers in Bioengineering and Biotechnology, 8, pp. 589094, 2020.

[22] Q. Yu, J. Tian. "Application of three-dimensional printing technique in manufacturing scaffolds for bone tissue engineering," Chinese Journal of Tissue Engineering Research, 19 (30), pp. 4870-4875, 2015.

[23] C.R. Rocha, A.R.T. Perez, D.A. Roberson, et al. "Novel ABS-based binary and ternary polymer blends for material extrusion 3D printing," Journal of Materials Research, 29 (17), pp. 1859-1866, 2015.

[24] R. Ikumi, T. Miyahara, N. Akino, et al. "Guided bone regeneration using a hydrophilic membrane made of unsintered hydroxyapatite and poly (L-lactic acid) in a rat bone-defect model," Dental Materials Journal, 37(6), pp. 912-918, 2018. 
[25] Y. Du, H. Liu, J. Shuang, et al. "Microsphere-based selective laser sintering for building macroporous bone scaffolds with controlled microstructure and excellent biocompatibility," Colloids and Surfaces B: Biointerfaces, 135, pp. 81-89, 2015.

[26] X. Zhou, G. Zhou, J. Radoslaw, et al. "Fabrication of polylactic acid (PLA)-based porous scaffold through the combination of traditional bio-fabrication and 3D printing technology for bone regeneration," Colloids and Surfaces B: Biointerfaces, 197, pp. 111420, 2020.

[27] M.C. Chen, H.C. Chiu, P.J. Kuo, et al. "Bone formation with functionalized $3 \mathrm{D}$ printed poly- $\varepsilon$-caprolactone scaffold with plasma-rich-fibrin implanted in critical-sized calvaria defect of rat," Journal of Dental Sciences, 16 (4), pp. 1055-1338, 2021.

[28] X. Yang, Y. Wang, Y. Zhou, et al. "The Application of Polycaprolactone in Three-Dimensional Printing Scaffolds for Bone Tissue Engineering," Polymers, 13 (16), pp. 2754-2754, 2021.

[29] J.M. Foletti, N. Lari, P. Dumas, et al. "PEEK customized implant for skull esthetic reconstruction," Revue de Stomatologie et de Chirurgie Maxillo-faciale, 113 (6), pp. 468-471, 2012.

[30] P. Scolozzi. "Maxillofacial reconstruction using polyetheretherketone patient-specific implants by "mirroring" computational planning," Aesthetic Plast Surg, 36 (3), pp. 660-665, 2012.

[31] L. Deng, Y. Deng, K. Xie. "AgNPs-decorated 3D printed PEEK implant for infection control and bone repair," Colloids Surf B Biointerfaces, 160, pp. 483-492, 2017.

[32] X. Feng, L. Ma, H. Liang, et al. "Osteointegration of 3D-Printed Fully Porous Polyetheretherketone Scaffolds with Different Pore Sizes," ACS Omega, 5 (41), pp. 26655-26666, 2020.

[33] M. Shie, W. Chang, L. Wei, et al. "3D Printing of Cytocompatible Water-Based Light-Cured Polyurethane with Hyaluronic Acid for Cartilage Tissue Engineering Applications," Materials, 10 (2), pp. 136-136, 2017.

[34] R.M. Day, V. Maquet, A.R. Boccaccini, et al. "In vitro and in vivo analysis of macroporous biodegradable poly (D, L-lactide-co-glycolide) scaffolds containing bioactive glass," Journal of biomedical materials research Part A, 75 (4), pp. 778-787, 2005.

[35] C. Tu. "Study on biocompatibility of a macroporouscomposite scaffold built by 3D printer," Huazhong University of Science and Technology, China, 2016.

[36] R. Socrates, S. Nagarajan, H. Belaid, et al. "Fabrication of 3D printed antimicrobial polycaprolactone scaffolds for tissue engineering applications," Materials Science and Engineering: C, 118, pp. 111525, 2021.

[37] X. Han, M. Sun, B. Chen, et al. "Lotus seedpod- inspired internal vascularized 3D printed scaffold for bone tissue repair," Bioactive materials, 6 (6), pp. 1639-1652, 2021. 